\title{
The damping of transverse oscillations of prominence threads: a comparative study
}

\author{
Roberto Soler, Ramon Oliver, and Jose Luis Ballester \\ Departament de Física, Universitat de les Illes Balears, E-07122 Palma de Mallorca, Spain \\ email: roberto.soler@uib.es
}

\begin{abstract}
Transverse oscillations of thin threads in solar prominences are frequently reported in high-resolution observations. The typical periods of the oscillations are in the range of 3 to $20 \mathrm{~min}$. A peculiar feature of the oscillations is that they are damped in time, with short damping times corresponding to few periods. Theoretically, the oscillations are interpreted as kink magnetohydrodynamic waves. However, the mechanism responsible for the damping is not well known. Here we perform a comparative study between different physical mechanisms that may damp kink waves in prominence threads. The considered processes are thermal conduction, cooling by radiation, resonant absorption, and ion-neutral collisions. We find that thermal conduction and radiative cooling are very inefficient for the damping of kink waves. The effect of ion-neutral collisions is minor for waves with periods usually observed. Resonant absorption is the only process that produces an efficient damping. The damping times theoretically predicted by resonant absorption are compatible with those reported in the observations.
\end{abstract}

Keywords. Sun: oscillations - Sun: corona - Sun: prominences - Sun: filaments - Waves

\section{Introduction}

Oscillations of small amplitude are frequently observed in solar prominences (see reviews by, e.g., Oliver \& Ballester 2002, Engvold 2008, Arregui et al. 2012). High-resolution observations often show that individual prominence threads, i.e., the building blocks of prominences, oscillate transversely (e.g., Lin et al. 2005, 2007, 2009, Okamoto et al. 2007, Ning et al. 2009). Some features of transverse thread oscillations are that the periods are in the range of 3 to $20 \mathrm{~min}$, that the velocity amplitudes are lower than $3 \mathrm{~km} \mathrm{~s}^{-1}$, and that the oscillations are quickly damped. Typically, the oscillation amplitude decreases to the noise level in less than 10 periods (Ning et al. 2009).

Transverse thread oscillations are theoretically interpreted as kink magnetohydrodynamic (MHD) waves propagating along the magnetic flux tube that supports the thread (e.g., Díaz et al. 2002, Terradas et al. 2008, Lin et al. 2009, Soler et al. 2010). However, the mechanism responsible for the damping is not well known and several possible physical processes have been suggested (e.g., Ballai 2003, Arregui et al. 2008, Oliver 2009). Here, we compare the efficiency of various mechanisms that may damp kink MHD waves in prominence threads, namely radiative cooling, thermal conduction, ion-neutral collisions, and resonant absorption. This brief work is based on the results extensively discussed in Soler (2010).

\section{Model and method}

The prominence thread model is schematically represented in Figure 1. It is composed of a straight cylindrical magnetic flux tube of radius $a$, filled with prominence plasma with density $\rho_{\mathrm{i}}$ and temperature $T_{\mathrm{i}}$, and embedded in a coronal environment with density $\rho_{\mathrm{e}}$ and temperature $T_{\mathrm{e}}$. Between the prominence and coronal plasma there is a transverse 


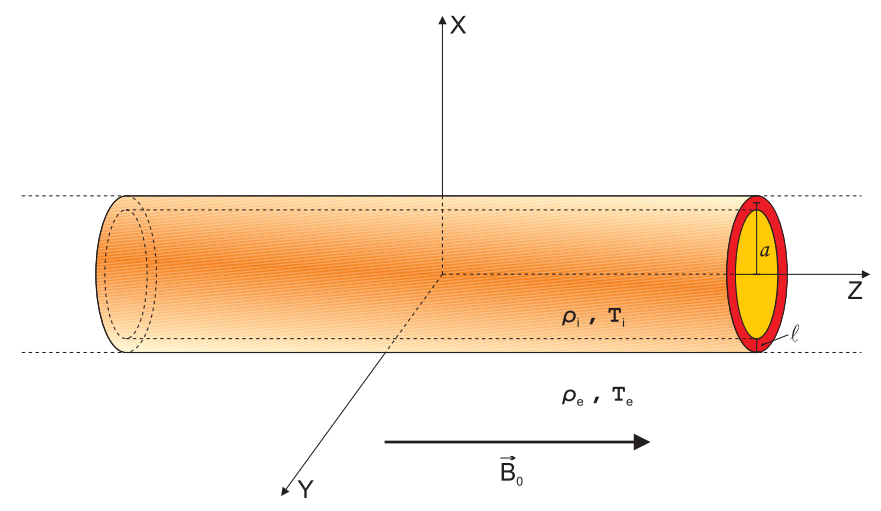

Figure 1. Schematic representation of the prominence thread model used in this work.

transitional layer of thickness $l$, where the physical conditions change continuously. The magnetic field strength, $B_{0}$, is constant. We use the following values for the various physical parameters: $\rho_{\mathrm{i}}=5 \times 10^{-11} \mathrm{~kg} \mathrm{~m}^{-3}, T_{\mathrm{i}}=8000 \mathrm{~K}, \rho_{\mathrm{e}}=2.5 \times 10^{-13} \mathrm{~kg} \mathrm{~m}^{-3}$, $T_{\mathrm{e}}=10^{6} \mathrm{~K}, a=100 \mathrm{~km}$, and $B_{0}=5 \mathrm{G}$. These parameters verify the pressure balance condition at the boundary of the thread. The thickness of the transitional layer, $l$, is considered a free parameter. The prominence plasma is assumed to be partially ionized and the ionization degree is a free parameter as well.

We study linear MHD waves superimposed on the static equilibrium. The governing equations for nonadiabatic perturbations are

$$
\begin{aligned}
\frac{\partial \rho^{\prime}}{\partial t}= & -\mathbf{v} \cdot \nabla \rho-\rho \nabla \cdot \mathbf{v}, \\
\rho \frac{\partial \mathbf{v}}{\partial t}= & -\nabla p^{\prime}+\frac{1}{\mu}\left[\left(\nabla \times \mathbf{B}^{\prime}\right) \times \mathbf{B}_{0}\right], \\
\frac{\partial p^{\prime}}{\partial t}= & -\gamma p \nabla \cdot \mathbf{v}-(\gamma-1)\left[\rho \frac{\partial L}{\partial \rho} \rho^{\prime}+\rho \frac{\partial L}{\partial T} T^{\prime}-\kappa_{\|} \frac{\partial^{2} T^{\prime}}{\partial z^{2}}\right], \\
\frac{\partial \mathbf{B}^{\prime}}{\partial t}= & \nabla \times\left(\mathbf{v} \times \mathbf{B}_{0}\right)-\nabla \times\left(\eta \nabla \times \mathbf{B}^{\prime}\right) \\
& +\nabla \times\left\{\frac{\eta_{\mathrm{C}}-\eta}{B_{0}^{2}}\left[\left(\nabla \times \mathbf{B}^{\prime}\right) \times \mathbf{B}_{0}\right] \times \mathbf{B}_{0}\right\}, \\
\frac{p^{\prime}}{p}= & \frac{\rho^{\prime}}{\rho}+\frac{T^{\prime}}{T},
\end{aligned}
$$

where $\rho, p, T, \mathbf{B}_{0}$ are the equilibrium density, gas pressure, and temperature, $\rho^{\prime}, p^{\prime}$, $T^{\prime}, \mathbf{B}^{\prime}$ are their corresponding perturbations, $\mathbf{v}$ is the velocity perturbation, and $\mu$ is magnetic permittivity. In addition, $\partial L / \partial \rho$ and $\partial L / \partial T$ are the partial derivatives of the the heat-loss function $L$ with respect to density and pressure, $\eta$ and $\eta_{\mathrm{C}}$ are Ohm's and Cowling's resistivities, and $\kappa_{\|}$is the parallel thermal conductivity. We refer the reader to Soler (2010) for the expressions of these quantities.

The perturbations are put proportional to $\exp \left(i k_{z} z+i m \varphi-i \omega t\right)$, where $k_{z}$ and $m$ are the longitudinal and azimuthal wavenumbers $(m=1$ for kink waves) and $\omega$ is the frequency. After fixing $k_{z}$ and $m$, Equations (2.1)-(2.5) form an eigenvalue problem where $\omega$ is the eigenvalue. The eigenvalue problem is solved numerically with the PDE2D code (Sewell 2005). Due to the presence of damping mechanism, $\omega$ is complex. The period, $P$, and exponential damping time, $\tau_{\mathrm{D}}$, are computed from the real and imaginary parts of 

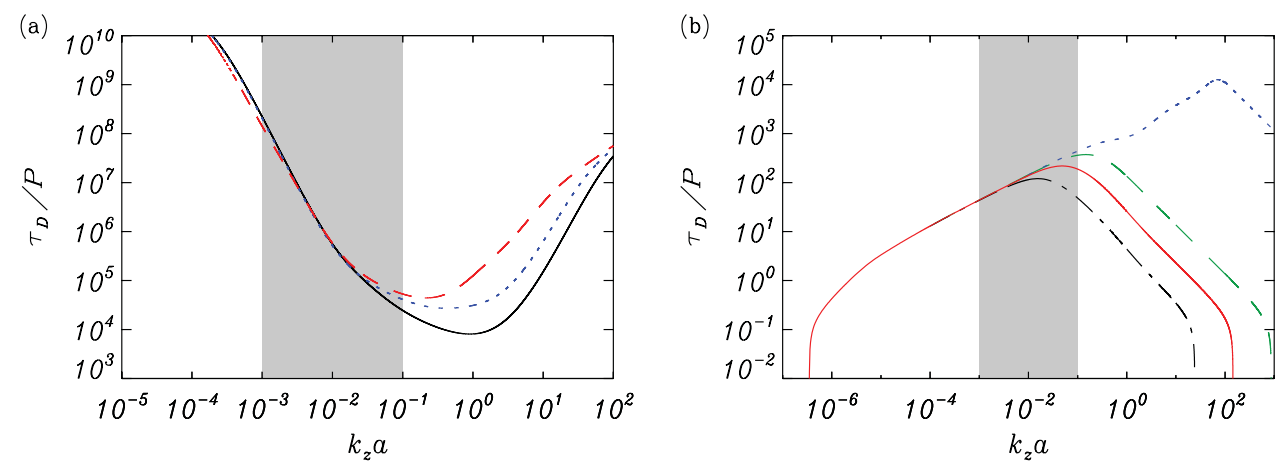

Figure 2. (a) Kink wave $\tau_{\mathrm{D}} / P$ vs. $k_{z} a$ for damping due to nonadiabatic effects. The various lines are for different parameterizations of the heat-loss function (see details in Soler 2010). (b) Same as panel (a) but for damping due to Cowling's and Ohm's diffusion. The various lines are for different ionization degrees from almost fully ionized (upper line) to almost fully neutral (lower line) thread. In both panels, the shaded area is the realistic range $k_{z} a \in\left[10^{-3}, 10^{-1}\right]$. Results for $l / a=0$.

$\omega$ as $P=2 \pi / \operatorname{Re}(\omega)$ and $\tau_{\mathrm{D}}=1 /|\operatorname{Im}(\omega)|$. Values of $\tau_{\mathrm{D}} / P \lesssim 10$ are needed for damping to be compatible with the observations.

\section{Results}

We compare the efficiency of the various damping mechanisms by computing the kink wave damping ratio, $\tau_{\mathrm{D}} / P$, produced by each process as function of the dimensionless wavenumber, $k_{z} a$, whose realistic values are in the interval $k_{z} a \in\left[10^{-3}, 10^{-1}\right]$.

Damping due to nonadiabatic effects. We compute the damping ratio when thermal conduction and radiative cooling are considered (Fig. 2a). We obtain $\tau_{\mathrm{D}} / P \gtrsim 10^{4}$, meaning that nonadiabatic effects are very inefficient damping mechanisms for kink waves and cannot explain the observed rapid attenuation.

Damping due to ion-neutral collisions. The effect of ion-neutral collisions is here included in the single-fluid approximation. Cowling's diffusion term in the induction equation (third term on the right-hand side of Equation (2.4)) contains the effect of collisions. For consistency, we also consider the effect of classic Ohm's diffusion. Figure 2b shows that $\tau_{\mathrm{D}} / P \gtrsim 10^{2}$ in the realistic range of $k_{z} a$, which points out that damping due to Cowling's and Ohm's diffusion is inefficient. Although efficient damping due to Ohm's/Cowling's diffusion is obtained for $k_{z} a$ smaller/larger than realistic values, this result has no relevance for the observed waves.

Damping due to resonant absorption. Resonant damping takes place in the nonuniform transitional layer where the global kink wave couples to local Alfvén and slow waves. An approximate expression for the kink mode $\tau_{\mathrm{D}} / P$ in the thin tube $\left(k_{z} a \ll 1\right)$ and thin boundary $(l / a \ll 1)$ approximations is (e.g., Ruderman \& Roberts 2002, Goossens et al. 2002)

$$
\frac{\tau_{\mathrm{D}}}{P} \approx \frac{2}{\pi} \frac{a}{l} \frac{\rho_{\mathrm{i}}+\rho_{\mathrm{e}}}{\rho_{\mathrm{i}}+\rho_{\mathrm{e}}}\left[1+\left(k_{z} a\right)^{2}\left(\frac{v_{\mathrm{s}}^{2}}{v_{\mathrm{s}}^{2}+v_{\mathrm{A}}^{2}}\right)^{2}\right]^{-1},
$$

where $v_{\mathrm{s}}$ and $v_{\mathrm{A}}$ are the sound and Alfvén velocities. The first term within the square brackets of Equation (3.1) is due to the Alfvén resonance and the second term is due to the slow resonance. For typical parameters the slow resonance can be neglected. Using $l / a=0.2$ and $k_{z} a=10^{-2}$, Equation (3.1) gives $\tau_{\mathrm{D}} / P \approx 3$, which suggests that 


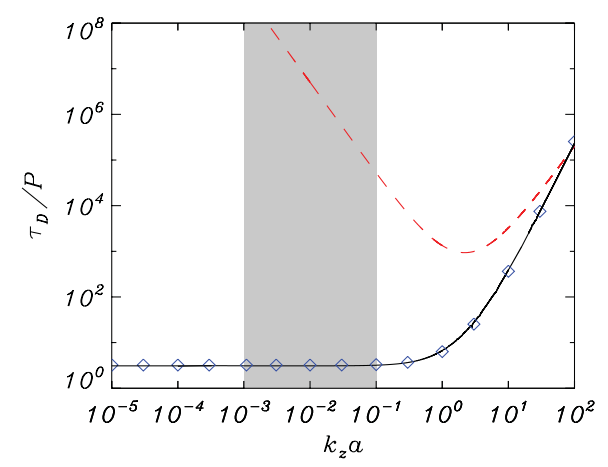

Figure 3. Same as Figure 2 but for damping due to resonant absorption. The solid line is the full result, the dashed line is the slow resonance damping, and symbols are the Alfvén resonance damping. We used $l / a=0.2$.

resonant absorption is an efficient damping mechanism. This result is confirmed by the full numerical computations displayed in Figure 3.

We conclude that, among the considered damping mechanisms, resonant coupling to Alfvén waves is the only process that produces kink wave damping times compatible with those observed.

\section{Acknowledgements}

We acknowledge support from MINECO and FEDER Funds through grant AYA201122846 and from CAIB through the 'grups competitius' program and FEDER Funds.

\section{References}

Arregui, I., Terradas, J., Oliver, R., \& Ballester, J. L. 2008, ApJL, 682, L141

Arregui, I., Oliver, R., \& Ballester, J. L. 2012, Living Rev. Sol. Phys., 9, 2

Ballai, I. 2003, $A \& A$, 410, L17

Díaz, A. J., Oliver, R., \& Ballester, J. L. 2002, ApJ, 580, 550

Engvold, O. 2008, Waves \& Oscillations in the Solar Atmosphere: Heating and MagnetoSeismology, Proceedings of the International Astronomical Union, IAU Symposium, 247, 152

Goossens, M., Andries, J., \& Aschwanden, M. J. 2002, A\&A, 394, L39

Lin, Y., Engvold, O., Rouppe van der Voort, L., Wiik, J E., \& Berger, T. E. 2005, Sol. Phys., 226,239

Lin, Y., Engvold, O., Rouppe van der Voort, L., \& van Noort, M. 2007, Sol. Phys., 246, 65

Lin, Y., Soler, R., Engvold, O., et al. 2009, ApJ, 704, 870

Ning, Z., Cao, W., Okamoto, T. J., Ichimoto, K., \& Qu, Z. Q. 2009, A\& A, 499, 595

Okamoto, T. J., Tsuneta, S., Berger, T. E., et al. 2007, Science, 318, 1577

Oliver, R. \& Ballester, J. L. 2002, Sol. Phys., 206, 45

Oliver, R. 2009, SSR, 149, 175

Ruderman, M. S. \& Roberts, B. 2002, ApJ, 577, 475

Sewell, G. 2005, The Numerical Solution of Ordinary and Partial Differential Equations, Pure and Applied Mathematics Series (New York: Wiley)

Soler, R., Arregui, I., Oliver, R., \& Ballester, J. L. 2010, ApJ, 722, 1778

Soler, R. 2010, PhD thesis, Universitat de les Illes Balears, available at http://www.uib.es/depart/dfs/Solar/thesis_roberto_soler.pdf

Terradas, J., Arregui, I., Oliver, R., \& Ballester, J. L. 2008, ApJL, 678, L153 